\title{
Eigenmode Analysis of a Light-Guiding Metal Line Loaded on a Dielectric Substrate Using the Imaginary-Distance Beam-Propagation Method
}

\author{
Jun Shibayama, Member, IEEE, Member, OSA, Tomohide Yamazaki, Junji Yamauchi, Member, IEEE, Member, OSA, \\ and Hisamatsu Nakano, Fellow, IEEE
}

\begin{abstract}
Fundamental characteristics of a light-guiding metal line are revealed and discussed through the eigenmode analysis using the three-dimensional (3-D) imaginary-distance beam-propagation method (ID-BPM) based on the alternating-direction implicit scheme. For the present ID-BPM, the multiplication factor of the eigenmode is derived and the paper described how the present method works in the ID procedure. An efficient absorbing boundary condition is described, which is suitable for the eigenmode analysis using the ID-BPM. After confirming the effectiveness of the present method, the characteristics of the light-guiding line composed of a metal (Au) with a finite width and thickness on a substrate $\left(\mathrm{SiO}_{2}\right)$ are investigated. Numerical results for a metal thickness of $0.2 \mu \mathrm{m}$ show that the effective index and the propagation loss decrease as the metal width is reduced. It is shown that not only the higher order modes but also the first mode has a cutoff metal width. Near the cutoff width, the propagation loss of the first mode $(\simeq 10 \mathrm{~dB} / \mathrm{mm}$ at a wavelength of $1.55 \mu \mathrm{m}$ ) is less than those of the higher order modes. Finally, in order to reduce the propagation loss, a dielectric core was added under the metal line.
\end{abstract}

Index Terms-Beam-propagation method (BPM), eigenmode analysis, imaginary-distance (ID) procedure, light-guiding metal line, surface plasmon-polaritons.

\section{INTRODUCTION}

I T IS KNOWN that a metal line can support surface plasmonpolaritons at optical wavelengths. This has attracted much attention, since the metal may be used as a light-guiding line in integrated optics. Recently, experimental and theoretical investigations have been made for a metal line embedded in a uniform dielectric material. In [1], the propagation characteristics of long-range plasmon-polariton waves were shown experimentally. The eigenmodes of the metal line were revealed by numerical analyses [2], [3].

Investigations have also been given to a metal line loaded on a dielectric substrate because of easiness of fabrication [4], [5]. The propagation losses were experimentally studied [4], and the

Manuscript received May 25, 2004; revised November 19, 2004. This work was supported in part by the Takahashi Industrial and Economic Research Foundation.

The authors are with the Faculty of Engineering, Hosei University, Tokyo 184-8584, Japan.

Digital Object Identifier 10.1109/JLT.2005.843449 surface plasmon-polariton waves were calculated using the finite-difference time-domain (FDTD) method [5]. There is, however, no theoretical discussion on fundamental characteristics, such as field profiles, effective indexes, and propagation losses, although the knowledge of these characteristics is indispensable for the use of a light-guiding line.

These characteristics are found by the eigenmode analysis. To calculate eigenmodes of optical waveguides, numerous methods have been proposed [6]. Of them, the imaginary-distance beampropagation method (ID-BPM) [7]-[10] has often been used as an efficient and convenient technique. Owing to its formulation with complex fields, complex modal fields of lossy waveguides are easily calculated [11]-[14]. The ID-BPM has the advantage that the calculated modal field is readily used as the input field for the propagating beam analysis. In other words, the eigenmode analysis followed by the propagating beam analysis can be performed by a single BPM algorithm. These facts motivate us to apply the ID-BPM to the eigenmode analysis of a metal line.

This paper describes the investigation of the fundamental characteristics of a metal $(\mathrm{Au})$ line loaded on a dielectric substrate $\left(\mathrm{SiO}_{2}\right)$ using the three-dimensional (3-D) ID-BPM based on the alternating-direction implicit (ADI) scheme. To the best of the authors' knowledge, this is the first application of the ID-BPM to the analysis of a light-guiding metal line.

For the ID-BPM presented in this paper, the multiplication factor of the eigenmode is derived and how the present method works in the ID procedure is described. To obtain the effective index, a technique is adopted for iteratively renewing the reference refractive index. An absorbing boundary condition (ABC) is derived from a modification to the perfectly matched layer (PML) [15], [16], which is suitable for the eigenmode analysis using the ID-BPM.

After demonstrating the superiority of the present method over the conventional one, the paper discusses the fundamental characteristics of a metal line at a wavelength of $1.55 \mu \mathrm{m}$. It is shown that a $3-\mu \mathrm{m}$-width line with a thickness of $0.2 \mu \mathrm{m}$ supports only a single mode and that an $11-\mu \mathrm{m}$ width supports three modes. The effective index and the propagation loss as a function of metal width is further evaluated. It is demonstrated that these two quantities decrease as the width of the line is reduced. Numerical results reveal that each mode has a cutoff 
width, e.g., the widths of the first three modes are estimated to be $\simeq 2.6,6.3$, and $10 \mu \mathrm{m}$. Near the cutoff width, the propagation loss of the first mode $(\simeq 10 \mathrm{~dB} / \mathrm{mm})$ is less than those of the higher order modes (losses of the second and third modes are $\simeq 15 \mathrm{~dB} / \mathrm{mm}$ and $15.5 \mathrm{~dB} / \mathrm{mm}$, respectively). Numerical results obtained from the 3-D BPM are also compared with the analytic solutions of a semi-infinite structure [2], [17]. It is shown from the comparison that the 3-D analysis is indispensable for the accurate evaluation of a line with a metal width of less than several micrometers.

In order to reduce the propagation loss, a dielectric core is finally added under the metal line. In this structure, a guided mode exists even below the cutoff width of the first mode for the metal line loaded on the dielectric substrate.

\section{NUMERICAL METHOD}

\section{A. Conventional ID-BPM}

Before describing the ID-BPM based on the ADI scheme, here we review the conventional ID-BPM based on the Crank-Nicolson (CN) scheme.

In the ID procedure, the propagation axis is taken to be the imaginary axis $\tau$. The basic equation of the 3-D semivectorial ID-BPM with the electric field $E$ for the quasi-transverse-magnetic (TM) mode is expressed as

$$
\frac{\partial E}{\partial \tau}=\frac{1}{\sigma}\left[D_{x x}+D_{y y}+\nu\right] E
$$

where

$$
\begin{aligned}
\sigma & =2 k n_{0} \\
\nu & =k^{2}\left(n^{2}-n_{0}^{2}\right) \\
D_{x x} E & =\frac{\partial^{2} E}{\partial x^{2}} \\
D_{y y} E & =\frac{\partial}{\partial y}\left(\frac{1}{n^{2}}\left(\frac{\partial}{\partial y}\left(n^{2} E\right)\right)\right)
\end{aligned}
$$

in which $k, n_{0}$ and $n$ are the free-space wavenumber, the reference refractive index, and the refractive index of the waveguide, respectively. Applying the $\mathrm{CN}$ scheme to (1), we have

$$
\begin{aligned}
{\left[1-\frac{\Delta \tau}{2 \sigma}\left(D_{x x}+D_{y y}+\nu\right)\right] E_{l+1} } & \\
= & {\left[1+\frac{\Delta \tau}{2 \sigma}\left(D_{x x}+D_{y y}+\nu\right)\right] E_{l} }
\end{aligned}
$$

where $l$ indicates the position along the propagation axis $\tau$. The improved three-point finite-difference formula [18] is employed to approximate the spatial derivatives in (2), i.e., $D_{x x} E_{(p, q)}$ is approximated by using $E_{(p-1, q)}, E_{(p, q)}$, and $E_{(p+1, q)}$, and $D_{y y} E_{(p, q)}$ is approximated using $E_{(p, q-1)}, E_{(p, q)}$, and $E_{(p, q+1)}$, where $p$ and $q$ represent the positions along the $x$ and $y$ axes, respectively. This approximation results in linear equations; each equation to be solved involves five unknowns. As a result, the system of equations becomes a broadly banded matrix.
Here, we derive the multiplication factor of the eigenmode field to see how the eigenmode field is amplified in the ID procedure. An arbitrary input field can be represented as a summation of the eigenmodes in the form

$$
E(x, y, \tau=0)=\sum a^{(i)} E^{(i)}(x, y)
$$

where $E^{(i)}$ and $a^{(i)}$ represent the $i$ th eigenmode field and its amplitude (although the summation should include both guided and radiation modes, we here deal with the guided modes only). Each eigenmode must satisfy the wave equation expressed as

$$
\left[D_{x x}+D_{y y}+k^{2}\left(n^{2}-\left(n_{e}^{(i)}\right)^{2}\right)\right] E^{(i)}=0
$$

where $n_{e}^{(i)}$ is the $i$ th effective index. Substituting (4) into (2), we obtain the following relation for the $i$ th eigenmode:

$$
E_{l+1}^{(i)}=\frac{1+\frac{\Delta \tau k^{2}}{2 \sigma}\left(\left(n_{e}^{(i)}\right)^{2}-n_{0}^{2}\right)}{1-\frac{\Delta \tau k^{2}}{2 \sigma}\left(\left(n_{e}^{(i)}\right)^{2}-n_{0}^{2}\right)} E_{l}^{(i)} \equiv A_{\mathrm{CN}}^{(i)} E_{l}^{(i)}
$$

As can be seen from (5), the $i$ th eigenmode field $E_{l}^{(i)}$ is amplified by the multiplication factor $A_{\mathrm{CN}}^{(i)}$ at each propagation step. In this case, the convergence of the $i$ th eigenmode field can be improved by a large $A_{\mathrm{CN}}^{(i)}$, which is obtained in such a way that the denominator of $A_{\mathrm{CN}}^{(i)}$ is chosen to be close to zero, i.e.,

$$
\Delta \tau \simeq \frac{2 \sigma}{k^{2}\left(\left(n_{e}^{(i)}\right)^{2}-n_{0}^{2}\right)}
$$

However, doing so requires the initial guess of $n_{e}^{(i)}$ close to the exact value that is unknown in practice. In addition, the resultant broadly banded matrix derived from (2) should be solved by iterative techniques such as the Bi-Conjugate Gradient Stabilized (Bi-CGSTAB) [19]. This is highly time consuming, as discussed subsequently in the paper.

\section{B. ADI-Based ID-BPM}

To efficiently solve (1), we apply the ADI scheme [19] to (2) so that

$$
\begin{aligned}
& {\left[1-\frac{\Delta \tau}{2 \sigma}\left(D_{x x}+\frac{\nu}{2}\right)\right]\left[1-\frac{\Delta \tau}{2 \sigma}\left(D_{y y}+\frac{\nu}{2}\right)\right] E_{l+1}} \\
& \quad=\left[1+\frac{\Delta \tau}{2 \sigma}\left(D_{x x}+\frac{\nu}{2}\right)\right]\left[1+\frac{\Delta \tau}{2 \sigma}\left(D_{y y}+\frac{\nu}{2}\right)\right] E_{l} .
\end{aligned}
$$

Introducing the intermediate field $E_{l+1 / 2}$, we obtain the following two-step algorithm:

$$
\begin{gathered}
{\left[1-\frac{\Delta \tau}{2 \sigma}\left(D_{x x}+\frac{\nu}{2}\right)\right] E_{l+1 / 2}=\left[1+\frac{\Delta \tau}{2 \sigma}\left(D_{y y}+\frac{\nu}{2}\right)\right] E_{l}} \\
{\left[1-\frac{\Delta \tau}{2 \sigma}\left(D_{y y}+\frac{\nu}{2}\right)\right] E_{l+1}=\left[1+\frac{\Delta \tau}{2 \sigma}\left(D_{x x}+\frac{\nu}{2}\right)\right] E_{l+1 / 2}}
\end{gathered}
$$

The spatial derivatives are approximated by the improved threepoint finite-difference formula, as discussed in Section II-A. It is worth mentioning that (8) and (9) maintain tridiagonal matrices, 
which can be solved by efficient techniques such as the Thomas algorithm.

For the ADI-based ID-BPM, the following relation for the $i$ th eigenmode is derived by expanding (7):

$$
E_{l+1}^{(i)}=\frac{1+\frac{\Delta \tau k^{2}}{2 \sigma}\left(\left(n_{e}^{(i)}\right)^{2}-n_{0}^{2}\right)+\epsilon}{1-\frac{\Delta \tau k^{2}}{2 \sigma}\left(\left(n_{e}^{(i)}\right)^{2}-n_{0}^{2}\right)+\epsilon} E_{l}^{(i)} \equiv A_{\mathrm{ADI}}^{(i)} E_{l}^{(i)}
$$

where

$$
\epsilon=\frac{\Delta \tau^{2}}{4 \sigma^{2}}\left(D_{x x}+\frac{\nu}{2}\right)\left(D_{y y}+\frac{\nu}{2}\right) .
$$

It should be noted that, in the multiplication factor $A_{\mathrm{ADI}}^{(i)}$, the additional term $\epsilon$ appears due to the use of the ADI scheme. Unlike (5), a large $A_{\mathrm{ADI}}^{(i)}$ for a specific mode cannot be determined, since the product of the second derivatives $D_{x x} D_{y y}$ in $\epsilon$ cannot be replaced with $n_{e}^{(i)}$ of (4).

As an alternative, we therefore employ a technique for iteratively renewing $n_{0}$ [10], [18]. In this technique, the effective index is calculated from

$$
k n_{e}=k n_{0}+\frac{\iint\left[\ln \left(E_{l+1}\right)-\ln \left(E_{l}\right)\right]\left|E_{l}\right|^{2} d x d y}{\Delta \tau \iint\left|E_{l}\right|^{2} d x d y}
$$

where the weighted average is introduced to minimize numerical errors [20], [21]. In the practical calculation, $n_{0}$ is updated using $n_{e}$ obtained at the previous step $l$. Since $n_{0}$ asymptotically converges to $n_{e}$ (without the initial guess of $n_{e}$ in (6)), $A_{\mathrm{ADI}}^{(i)}$ in (10) becomes unity, while eliminating the effect of $\epsilon$. After sufficiently long propagation, only the fundamental mode generates without being amplified and the other higher order modes decay (the higher order modes are also obtained using the Gram-Schmidt orthogonalization technique [9]). Because of $A_{\mathrm{ADI}}^{(i)} \simeq 1$, this approach appears to be less efficient than the conventional ID-BPM with a large $A_{\mathrm{CN}}^{(i)}$. However, as will be found subsequently in the paper, the present approach efficiently provides the effective index and propagation loss, since the central processing unit (CPU) time per single calculation step is substantially reduced when compared with the conventional ID-BPM.

\section{Absorbing Boundary Condition}

When using the conventional PML-ABC for the eigenmode analysis of a metal line, we have observed that the field tends to diverge. To overcome this problem, we make a modification to the PML-ABC.

In the conventional PML region, the second derivative in the $\alpha$ direction is transformed as follows [15]:

$$
\frac{\partial^{2}}{\partial \alpha^{2}} \rightarrow \frac{1}{s} \frac{\partial}{\partial \alpha}\left(\frac{1}{s} \frac{\partial}{\partial \alpha}\right)
$$

where

$$
\frac{1}{s}=\frac{1}{1+\Gamma^{2}}+j \frac{\Gamma}{1+\Gamma^{2}}
$$

in which $\alpha=x$ or $y$ and

$$
\Gamma=\frac{\sigma_{e}}{\omega \varepsilon_{0} n^{2}}
$$

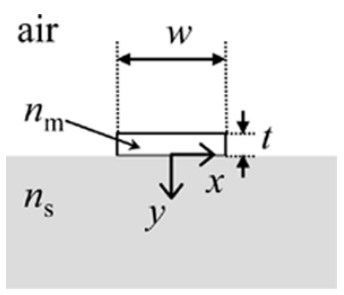

Fig. 1. Configuration of a metal line.

$$
\begin{aligned}
\sigma_{e} & =\sigma_{\max }\left(\frac{\rho}{d}\right)^{m} \\
\sigma_{\max } & =\frac{m+1}{2} \frac{\varepsilon_{0} c n^{2}}{d} \ln \frac{1}{R} .
\end{aligned}
$$

In these equations, $\omega$ is the angular frequency, $\varepsilon_{0}$ the permittivity of the free space, $c$ the speed of light in a vacuum, $\sigma_{e}$ the electric conductivity, $\sigma_{\max }$ the maximum conductivity, $\rho$ the distance from the interface between the computational and the PML regions, $d$ the thickness of the PML, $m$ the order of the polynomial, and $R$ the theoretical reflection coefficient.

In the eigenmode analysis using the ID-BPM, the field near the computational window edge is dominated by an evanescent wave, provided that the field is not a leaky wave. This leads to the fact that the phase change hardly occurs in the lateral directions. Therefore, when an $\mathrm{ABC}$ is imposed on the computational window edge, only the amplitude of the field should be reduced without affecting the phase. Based on this consideration, we adopt only the real part of (13), while eliminating the imaginary part (similar but not exactly the same treatment can be found in [13]). This ABC is no longer the PML-ABC, since the impedance matching condition is not satisfied, and the traveling wave cannot be absorbed ( $R$ in (13), which is analytically expressed by a complicated form [15], [16] for the conventional PML, is empirically determined in Section III-B). In this paper, nevertheless, this ABC is called a modified PML, since it is derived from a modification to the conventional PML. The effectiveness of the modified PML will be discussed in Section III.

\section{ANALYSis of Metal LiNES}

We treat the light-guiding metal line shown in Fig. 1. The refractive indexes of the metal $(\mathrm{Au})$ and substrate $\left(\mathrm{SiO}_{2}\right)$ are $n_{m}=0.18-j 10.2$ [22], [23] and $n_{s}=1.459$, respectively, at $\lambda=1.55 \mu \mathrm{m}$. Since the structure is asymmetrical with respect to the $x$ axis, only the asymmetrical mode (the so-called $a_{b}$ mode in [2]) is supported. In this paper, the conventional nomenclature for a dielectric waveguide, e.g., $E_{11}^{y}$, is employed for convenience.

\section{A. Preliminary Calculations of Two-Dimensional Structures}

Before analyzing a 3-D metal line, we preliminarily evaluate the characteristics of a two-dimensional (2-D) structure, i.e., $w=\infty$ in Fig. 1, in order to determine the thickness of the metal. Fig. 2 shows the effective index and the propagation loss as a function of thickness $t$ of the metal line, calculated using the 2-D ID-BPM. Note that the mode supported in this model is asymmetric. It is seen that these two quantities increase as the metal thickness becomes thin (this contrasts to the fact that these two quantities decrease for a symmetric mode [2]). Although not 


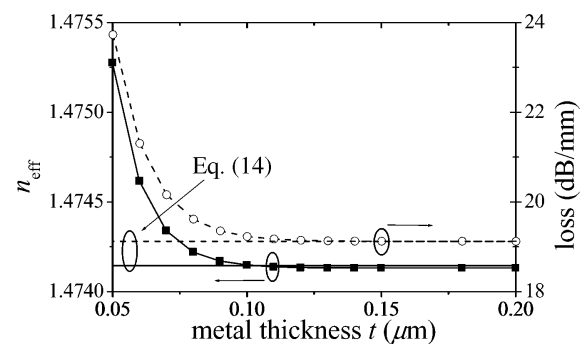

Fig. 2. Effective index and propagation loss for a 2-D structure.

illustrated, this is because the field for the asymmetric mode becomes more confined to the metal with a reduction in the metal thickness [2]. In addition, the increase in the metal thickness leads to the fact that the effective index and the propagation loss approach constant values, with the loss becoming smaller. These values for $t=0.2 \mu \mathrm{m}$ agree well with those obtained from the following analytic solution for a semi-infinite structure composed of a metal and a dielectric, which are indicated by the solid and dashed straight lines in Fig. 2 [2], [17]:

$$
\gamma=k \sqrt{\frac{n_{m}^{2} n_{s}^{2}}{n_{m}^{2}+n_{s}^{2}}} .
$$

The real and imaginary parts of (14) correspond to the phase and attenuation constants, respectively. As a result, since the metal with $t=0.2 \mu \mathrm{m}$ is thick enough to give the constant values of the effective index and the small propagation loss, we choose this thickness in the following 3-D analysis.

\section{B. Validity of the ADI-Based ID-BPM}

We discuss the validity of the ADI-based ID-BPM with the modified PML for the analysis of a 3-D metal line. As an example, the metal line with $t=0.2 \mu \mathrm{m}$ and $w=3 \mu \mathrm{m}$ is treated. Due to the symmetry of the metal line, only half the section is analyzed in the following analysis.

First, we check the effectiveness of the technique for iteratively renewing $n_{0}$. Fig. 3(a) and (b) shows the convergence behavior of the effective index and the propagation loss of the first mode $\left(E_{11}^{y}\right)$, respectively, obtained from (11) as a function of the number of propagation steps. In this analysis, the sampling widths are chosen to be $\Delta x=0.05 \mu \mathrm{m}$ and $\Delta y=0.01 \mu \mathrm{m}$, and the propagation step length $\Delta \tau=0.5 \mu \mathrm{m}$. The computational window edges are terminated with the modified PML: only the real part of (13) is used. The parameters in (13) are $d=0.5 \mu \mathrm{m}$, $m=3$, and $R=10^{-10}$. The computational region is set to be $0 \mu \mathrm{m}<x<15 \mu \mathrm{m}$ and $-1 \mu \mathrm{m}<y<4 \mu \mathrm{m}$ (a PML thickness of $d=0.5 \mu \mathrm{m}$ is included along the computational window edge). The initial value of $n_{0}$ is chosen to be $1,1.23$ and $n_{s}$. Once the field propagates $(\tau \geq \Delta \tau)$, the value of $n_{0}$ becomes complex. From Fig. 3, it can be found that the effective index and the propagation loss converge, regardless of the choice of $n_{0}$.

Here, we compare the efficiency of the present ADI-based ID-BPM with that of the conventional ID-BPM without the ADI scheme described in Section II-A. For the conventional ID-BPM, we substitute the effective index obtained in Fig. 3 into (6), where $n_{0}$ is set to be 1.4 , in order to obtain an appropriate parameter for fast convergence. The effective index

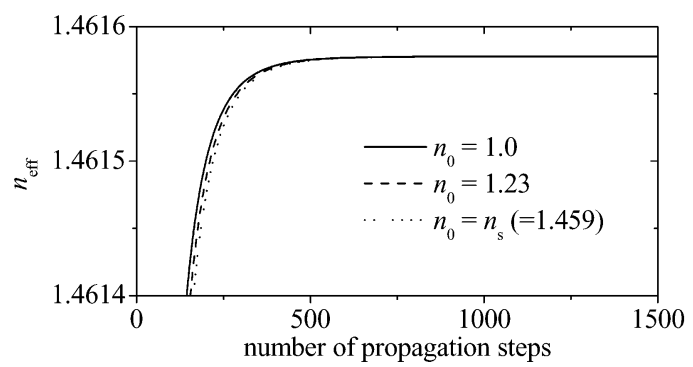

(a)

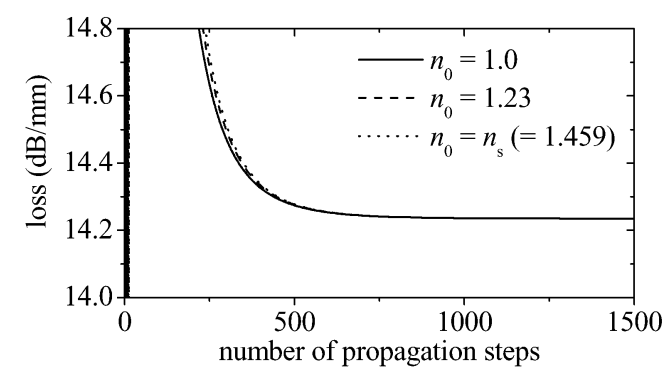

(b)

Fig. 3. (a) Effective index and (b) propagation loss as a function of propagation steps.

TABLE I

NuMBER OF PROPAGATION STEPS AND CPU TIME

\begin{tabular}{ccc}
\hline & ADI & conventional \\
\hline propagation steps & 950 & 4 \\
\hline CPU time $(\mathrm{sec})$ & 470 & 1460 \\
\hline
\end{tabular}

is calculated by the following variational expression without using the weak form:

$$
\left(k n_{e}\right)^{2}=\frac{\iint\left[k^{2} n^{2}|E|^{2}+E^{*}\left(D_{x x} E+D_{y y} E\right)\right] d x d y}{\iint|E|^{2} d x d y}
$$

where the superscript $*$ represents the complex conjugate. The real and imaginary parts of (15) correspond to the phase and attenuation constants, respectively.

Table I shows the number of propagation steps and the CPU time, which are required to obtain the converged values of the effective index and propagation loss (decibels per millimeter) up to six and two decimal places, respectively (the converged values obtained from (11) in the present ID-BPM are exactly the same as those from (15) in the conventional ID-BPM). The calculations are carried out on a 3.2-GHz PC. As found in Table I, the present ADI-based ID-BPM yields a substantial reduction in CPU time, although it requires a number of propagation steps. For the conventional ID-BPM, the long CPU time results from the solution of a broadly banded matrix. Further calculation using the conventional ID-BPM reveals that a slight deviation from the exact effective index gives rise to slow convergence: 39 steps and $3189 \mathrm{~s}$ are required when using an effective index of 5\% larger than that in Fig. 3. As a result, the ADI-based ID-BPM efficiently provides the numerical results without any knowledge of the effective index, while the conventional ID-BPM requires knowledge of the exact effective index.

Next, we discuss the performance of the modified PML. Since the $E_{11}^{y}$ field for $w=3 \mu \mathrm{m}$ extends toward the substrate as 


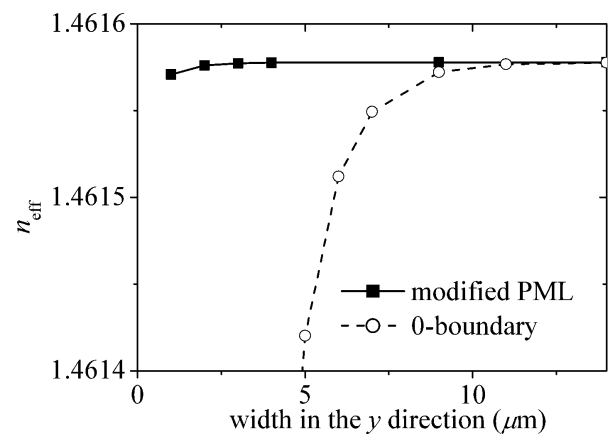

(a)

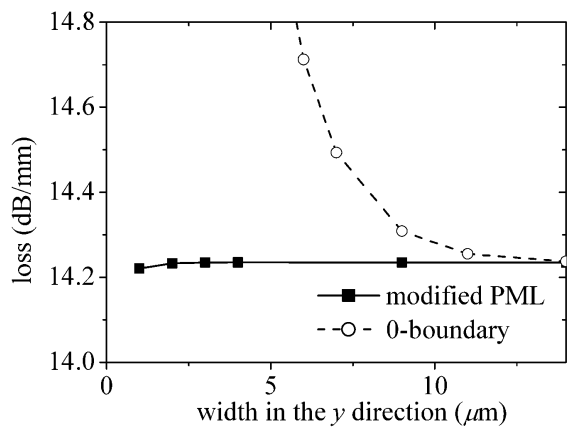

(b)

Fig. 4. (a) Effective index and (b) propagation loss as a function of width in the $y$ direction.

will be shown in Fig. 5(a), the width of the computational region in the $y$ direction affects the accuracy of the numerical results. Therefore, we investigate to what extent the computational region in the $y$ direction can be reduced when using the modified PML, where the width of the $x$ direction is fixed to be $0 \mu \mathrm{m}<x<15 \mu \mathrm{m}$. Fig. 4(a) and (b) shows the effective index and the propagation loss, respectively, as a function of width in the $y$ direction that includes a PML thickness of $d=0.5 \mu \mathrm{m}$. It is noteworthy that for the modified PML, the width in the $y$ direction can be reduced to $3 \mu \mathrm{m}$. For reference, also included in Fig. 4 are the results obtained using the zero boundary condition. In this case, the width should be $14 \mu \mathrm{m}$ to obtain the same results as those calculated using the modified PML.

\section{Characteristics of Three-Dimensional Structures}

Since the validity of the present ADI-based ID-BPM has been demonstrated, the characteristics of the metal line are investigated in detail. Fig. 5 shows the eigenmode fields of the TM mode $\left(E^{y}\right)$ for $w=3 \mu \mathrm{m}$ and $11 \mu \mathrm{m}$. As illustrated in Fig. 5, the metal line for $w=3 \mu \mathrm{m}$ supports only $E_{11}^{y}$, while that for $w=11 \mu \mathrm{m}$ supports $E_{11}^{y}, E_{21}^{y}$ and $E_{31}^{y}$.

To investigate the mode field profile around the interface in detail, we illustrate in Fig. 6 the cross section of the field distributions $\left(E_{11}^{y}\right)$ for $w=3 \mu \mathrm{m}$ and $11 \mu \mathrm{m}$, observed at $x=\Delta x / 2$. It can be seen that the field is localized around the interface. For reference, also included is the field for the 2-D structure, i.e., $w=\infty$, which is calculated by the 2-D ID-BPM. The field for $w=11 \mu \mathrm{m}$ is almost the same as that for $w=\infty$. When compared with the field for $w=11 \mu \mathrm{m}$, the field for $w=3 \mu \mathrm{m}$ extends further into the substrate.

We next evaluate how the effective index and the propagation loss depend on the width of the metal line. Fig. 7(a) and
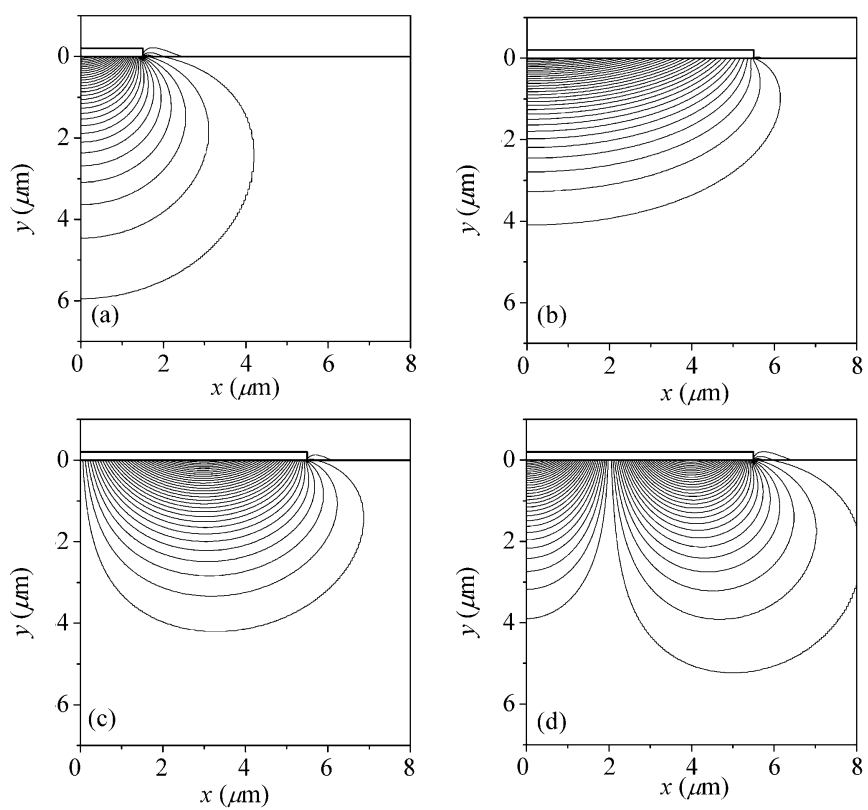

Fig. 5. Field distributions: (a) $E_{11}^{y}$ for $w=3 \mu \mathrm{m}$, (b) $E_{11}^{y}$ for $w=11 \mu \mathrm{m}$, (c) $E_{21}^{y}$ for $w=11 \mu \mathrm{m}$, and (d) $E_{31}^{y}$ for $w=11 \mu \mathrm{m}$. Only half the section $(x>0)$ is shown. Although the computational region can be reduced using the modified PML, it is taken to be large enough to show the mode profile.

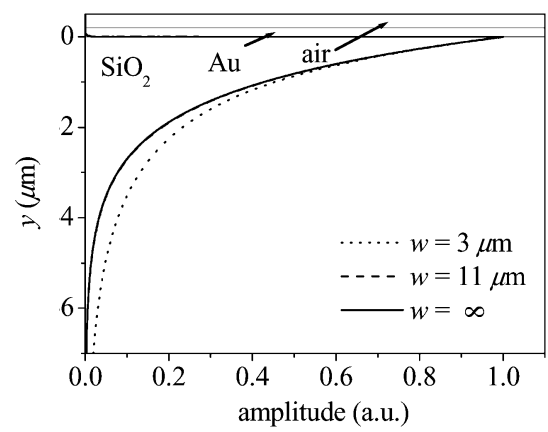

Fig. 6. Field distributions $(x=\Delta x / 2)$.

(b) shows the effective index and the propagation loss, respectively. It is seen that these two quantities decrease as $w$ becomes small. Careful examination of the results shows that $E_{11}^{y}, E_{21}^{y}$ and $E_{31}^{y}$ are cut off at $w \simeq 2.6,6.3$, and $10 \mu \mathrm{m}$, respectively. This fact contrasts with the symmetrical mode observed for a metal line embedded in a uniform dielectric material [2], [3]. Near the cutoff width, the loss of $E_{11}^{y}$ is smaller than those of the higher order modes. The decrease of the propagation loss is attributed to the reduced metal region that absorbs the field and the resultant extended field toward the substrate. The extension of the field also leads to the fact that the effective index approaches the refractive index of the substrate $\left(n_{s}=1.459\right)$, as shown in Fig. 7(a).

Note, for a specific metal width, that the propagation loss of the higher order mode is smaller than that of the lower order mode. One reason of the small loss stems from the extended field of the higher order mode, when compared with that of the lower order mode.

In Fig. 7(a) and (b), the results for $w=\infty$ obtained in Section III-A are also included. As expected from the agreement between the fields for $w=11 \mu \mathrm{m}$ and $w=\infty$ in Fig. 6, the propagation loss of the former is close to that of the latter. For 


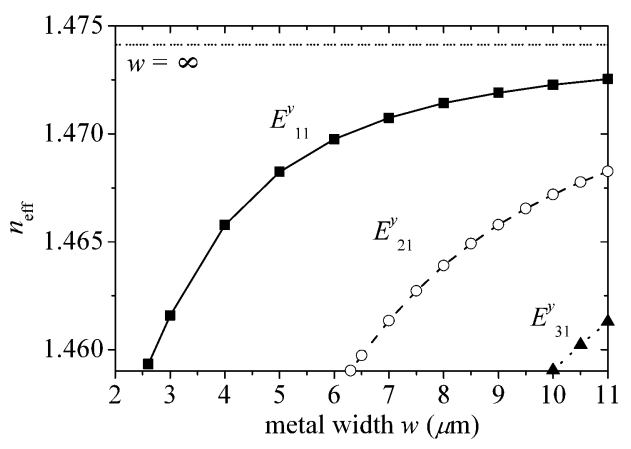

(a)

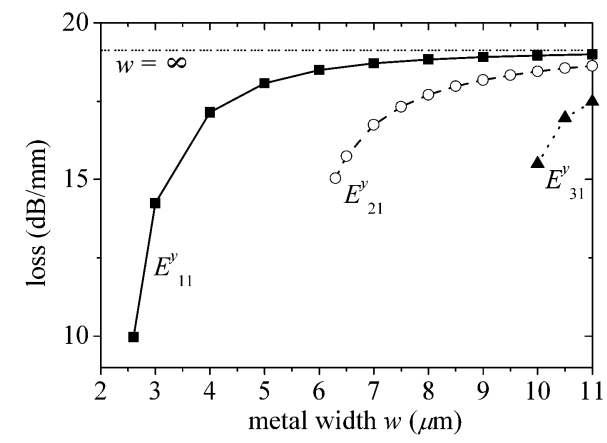

(b)

Fig. 7. (a) Effective index and (b) propagation loss.

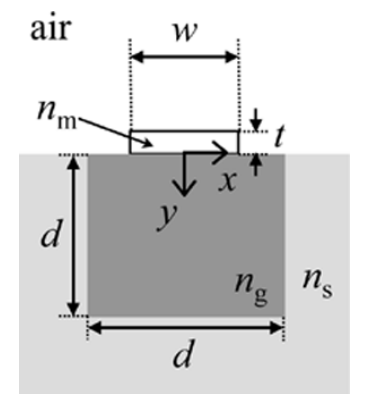

Fig. 8. Configuration of a metal line with an embedded dielectric core.

a narrow metal width, however, the results of the 3-D structure deviate from those of the 2-D one. Also note that the results of the 2-D structure cannot predict the characteristics of the higher order modes. As a result, the 3-D analysis is indispensable for the accurate evaluation of a metal line with a width of less than several micrometers.

\section{Reduction in the Propagation Loss}

Although the metal line loaded on the substrate is easy to fabricate, its propagation loss is larger than that of the metal line embedded in a uniform dielectric material. To reduce the propagation loss, we add a dielectric core $(d=3 \mu \mathrm{m})$ under the metal line, as shown in Fig. 8. The refractive index $n_{g}$ is chosen to be 1.5 , in which the dielectric waveguide without the metal $(w=0 \mu \mathrm{m})$ supports only a single mode at $\lambda=1.55 \mu \mathrm{m}$.

Fig. 9 illustrates the cross section of the field distributions for several values of $w$, observed at $x=\Delta x / 2$. It is seen that, as $w$ is reduced, the mode gradually changes from the surface

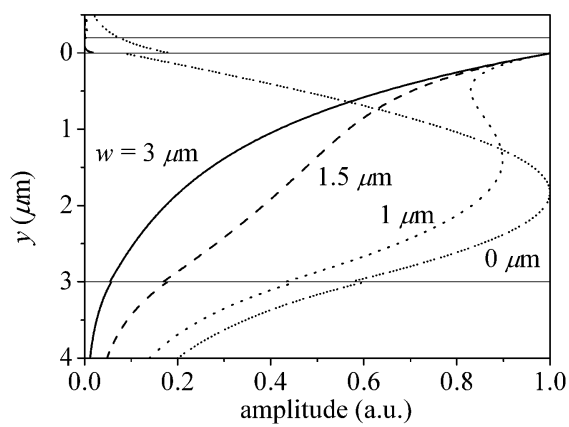

Fig. 9. Field distributions $(x=\Delta x / 2)$.

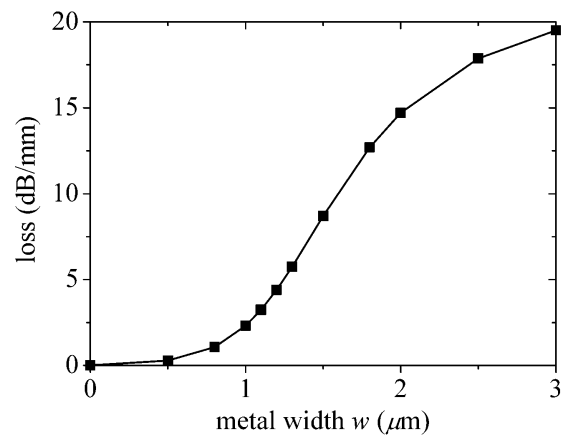

Fig. 10. Propagation loss as a function of $w$.

plasma-like mode to the guided mode of the dielectric waveguide. The localization of the field around the interface is observed for $w$ down to $\simeq 1.3 \mu \mathrm{m}$.

Note that for the metal line with the embedded core, a mode exists even below the cutoff width $(2.6 \mu \mathrm{m})$ of the first mode for the metal line loaded on the dielectric substrate discussed in Section III-C. Therefore, it is expected that the propagation loss should be reduced for a narrow metal width. Fig. 10 depicts the propagation loss as a function of $w$. As can be seen, the propagation loss reduces with a reduction in $w$. For $w=1.3 \mu \mathrm{m}$ and $1.5 \mu \mathrm{m}$, the propagation losses are calculated to be 5.6 and $8.7 \mathrm{~dB} / \mathrm{mm}$, respectively. These values are smaller than that of $10 \mathrm{~dB} / \mathrm{mm}$ for the metal line with $w=2.6 \mu \mathrm{m}$ on the uniform dielectric substrate.

\section{CONCLUSION}

This paper discussed the eigenmode of the light-guiding metal line loaded on a dielectric substrate using the ADI-based ID-BPM. It is confirmed that the present method efficiently offers the complex mode of the metal line. For the metal line on the substrate, it is found that the effective index and the propagation loss decrease with a reduction in the metal width. The decrease of the propagation loss is attributed to the reduced metal region that absorbs the field and the resultant extended field toward the substrate. A comparison of the results obtained from the 3-D BPM and the analytic solution of the semi-finite structure shows that the 3-D analysis is required for the accurate evaluation of a light-guiding line with a metal width of less than several micrometers. To reduce the propagation loss, a dielectric core under the metal line was added.

Throughout this paper, the semivectorial BPM was employed, which ignores mixed derivatives in coupled-wave equations. The full-vectorial analysis is left for a future study. 


\section{ACKNOWLEDGMENT}

The authors would like to thank $\mathrm{H}$. Aono, who provided the data on Figs. 9 and 10.

\section{REFERENCES}

[1] R. Charbonneau, P. Berini, E. Berolo, and E. Lisicka-Shrzek, "Experimental observation of plasmon-polariton waves supported by a thin metal film of finite width," Opt. Lett., vol. 25, no. 11, pp. 844-846, 2000.

[2] P. Berini, "Plasmon-polariton waves guided by thin lossy metal films of finite width: Bound modes of symmetric structures," Phys. Rev. B, Condens. Matter, vol. 61, no. 15, pp. 10484-10 503, 2000.

[3] S. J. Al-Bader, "Optical transmission on metallic wires-fundamental modes," IEEE J. Quantum Electron., vol. 40, no. 3, pp. 325-329, Mar. 2004

[4] Y. Katagiri, Y. Nakano, I. Kobayashi, Y. Mitsuoka, H. Shinojima, H. Fukuda, and T. Goto, "Propagation characteristics of surface-plasmon polariton waves along two-dimensional metal patterns," in Contemporary Photonics Technology 2002, Conf. Proc., vol. E-30, Tokyo, Japan, 2002, pp. 119-120.

[5] H. Fukuda, H. Shinojima, T. Goto, and Y. Katagiri, "The analysis of surface plasmon polariton waves using FDTD method," in Proc. IEICE Soc. Conf., vol. C-3-74, 2002, pp. 174-174.

[6] R. Scarmozzino, A. Gopinath, R. Pregla, and S. Helfert, "Numerical techniques for modeling guided-wave photonics devices," IEEE J. Sel. Topics Quantum Electron., vol. 6, no. 1, pp. 150-162, Jan.-Feb. 2000.

[7] D. Yevick and W. Bardyszewski, "Correspondence of variational finitedifference (relaxation) and imaginary-distance propagation methods for modal analysis," Opt. Lett., vol. 17, no. 5, pp. 329-330, 1992.

[8] C. L. Xu, W. P. Huang, and S. K. Chaudhuri, "Efficient and accurate vector mode calculations by beam propagation method," J. Lightw. Technol., vol. 11, no. 7, pp. 1209-1215, Jul. 1993.

[9] J. C. Chen and S. Jüngling, "Computation of higher-order waveguide modes by the imaginary-distance beam propagation method," Opt. Quantum Electron., vol. 29, no. 3, pp. S199-S205, 1994.

[10] J. Shibayama, M. Sekiguchi, J. Yamauchi, and H. Nakano, "Eigenmode analysis of optical waveguides by an improved finite-difference imaginary-distance beam propagation method," Electron. Comm. in Japan, pt. 2, vol. 81, no. 4, pp. 1-9, 1998.

[11] S. Jüngling and J. C. Chen, "Imaginary-distance beam propagation for passive and active waveguide structures," presented at the Progress in Electromagnetic Research Symposium, Seattle, WA, Jul. 24-28, 1995.

[12] Y. Tsuji and M. Koshiba, "Guided-mode and leaky-mode analysis by imaginary distance beam propagation method based on finite element scheme," J. Lightw. Technol., vol. 18, no. 4, pp. 618-623, Apr. 2000.

[13] K. Saitoh and M. Koshiba, "Full-vectorial imaginary-distance beam propagation method based on a finite element scheme: Application to photonic crystal fibers," IEEE J. Quantum Electron., vol. 38, no. 7, pp. 927-933, Jul. 2002.

[14] S. S. A. Obayya, B. M. A. Rahman, K. T. V. Grattan, and H. A. El-Mikati, "Full vectorial finite-element-based imaginary distance beam propagation solution of complex modes in optical waveguides," J. Lightw. Technol., vol. 20, no. 6, pp. 1054-1059, Jun. 2002.

[15] W. P. Huang, C. L. Xu, W. Lui, and K. Yokoyama, "The perfectly matched layer (PML) boundary condition for the beam propagation method," IEEE Photon. Technol. Lett., vol. 8, no. 5, pp. 649-651, May 1996.

[16] D. Yevick, J. Yu, and F. Schmidt, "Analytic studies of absorbing and impedance-matched boundary layers," IEEE Photon. Technol. Lett., vol. 9, no. 1, pp. 73-75, Jan. 1997.

[17] J. J. Burke, G. I. Stageman, and T. Tamir, "Surface-polariton-like waves guided by thin, lossy metal films," Phys. Rev. B, Condens. Matter, vol. 33, no. 8, pp. 5186-5201, 1986.

[18] J. Yamauchi, Propagating Beam Analysis of Optical Waveguides. Baldock, Hertfordshire, U.K.: Research Studies Press, 2003.

[19] J. Shibayama, A. Yamahira, T. Mugita, J. Yamauchi, and H. Nakano, "A finite-difference time-domain beam-propagation method for TE- and TM-wave analyses," J. Lightw. Technol., vol. 21, no. 7, pp. 1709-1715, Jul. 2003.

[20] S. M. Lee, "Finite-difference vectorial-beam-propagation method using Yee's discretization scheme for modal fields," J. Opt. Soc. Amer. A, Opt. Image Sci., vol. 13, no. 7, pp. 1369-1377, 1996.

[21] T. Ando, H. Nakayama, S. Numata, J. Yamauchi, and H. Nakano, "Eigenmode analysis of optical waveguides by a Yee-mesh-based imaginary-distance propagation method for an arbitrary dielectric interface," J. Lightw. Technol., vol. 20, no. 8, pp. 1627-1634, Aug. 2002.
[22] M. A. Ordal, L. L. Long, R. J. Bell, S. E. Bell, R. R. Bell, R. W Alexander, Jr, and C. A. Ward, "Optical properties of the metals Al, $\mathrm{Co}, \mathrm{Cu}, \mathrm{Au}, \mathrm{Fe}, \mathrm{Pb}, \mathrm{Ni}, \mathrm{Pd}, \mathrm{Pt}, \mathrm{Ag}, \mathrm{Ti}$, and $\mathrm{W}$ in the infrared and far infrared," Appl. Opt., vol. 22, no. 7, pp. 1099-1119, 1983.

[23] M. Rajarajan, C. Themistos, B. M. A. Rahaman, and K. T. V. Grattan, "Characterization of metal-clad TE/TM mode splitters using the finite element method," J. Lightw. Technol., vol. 15, no. 12, pp. 2264-2269, Dec. 1997.

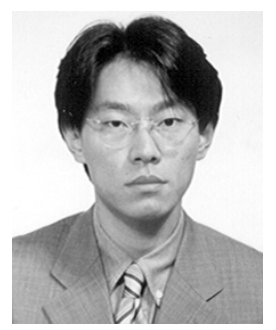

Jun Shibayama (M'03) was born in Kashiwa, Japan, on July 1, 1969. He received the B.E., M.E., and Dr.E. degrees from Hosei University, Tokyo, Japan, in 1993, 1995, and 2001, respectively.

In 1995, he joined Opto-Technology Laboratory, Furukawa Electric Compnay, Ltd., Ichihara, Japan. Since 1999, he has been an Assistant at Hosei University. His research interests include numerical analysis of optical waveguides.

Dr. Shibayama is a Member of the Optical Society of America (OSA) and the Institute of Electronics, Information and Communication Engineers (IEICE) of Japan.

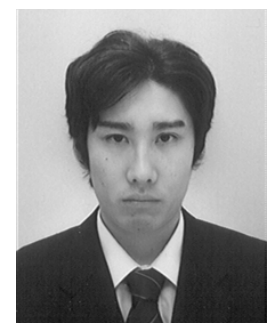

Tomohide Yamazaki was born in Tokyo, Japan, on May 1, 1979. He received the B.E. degree from Hose University, Tokyo, Japan, in 2003, where is currently working toward the M.E. degree.

Mr. Yamazaki is a Member of the Institute of Electronics, Information and Communication Engineers (IEICE) of Japan.

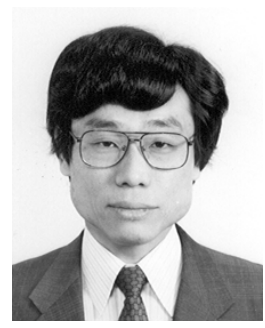

Junji Yamauchi (M'85) was born in Nagoya, Japan, on August 23, 1953. He received the B.E., M.E., and Dr.E. degrees from Hosei University, Tokyo, Japan, in 1976,1978 , and 1982, respectively.

From 1984 to 1988 , he served as a Lecturer in the Electrical Engineering Department of Tokyo Metropolitan Technical College. Since 1988, he has been a member of the faculty of Hosei University, where he is now a Professor of Electronic Informatics. His research interests include optical waveguides and circularly polarized antennas. $\mathrm{He}$ is the author of Propagating Beam Analysis of Optical Waveguides (Baldock, Hertfordshire, U.K.: Research Studies Press, 2003)

Dr. Yamauchi is a Member of the Optical Society of America (OSA) and the Institute of Electronics, Information and Communication Engineers (IEICE) of Japan.

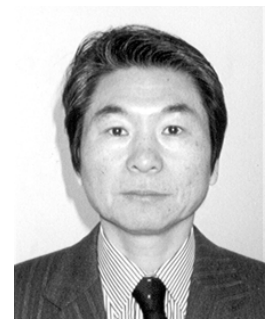

Hisamatsu Nakano (M'75-SM'87-F'92) was born in Ibaraki, Japan, on April 13, 1945. He received his B.E., M.E., and Dr.E. degrees in electrical engineering from Hosei University, Tokyo, Japan, in 1968, 1970, and 1974, respectively.

Since 1973, he has been a member of the faculty of Hosei University, where he is now a Professor of Electronic Informatics. His research topics include numerical methods for low- and high-frequency antennas and optical waveguides. He has published more than 190 refereed journal papers, more than 150 international symposium papers, and more than 500 national symposium papers. He is the author of a book titled Helical and Spiral Antennas (New York: Research Studies Press, Wiley, 1987) and the coauthor of Analysis Methods of Electromagnetic Wave Problems, Volume Two (Norwood, MA: Artech House, 1986). In addition, he is the author of Helical and Spiral Antennas, Encyclopedia of Communications (New York: Wiley, 2002).

Prof. Nakano received the Institution of Electrical Engineers (IEE) International Conference on Antennas and Propagation Best Paper Award and the IEEE TRANSACTIONS ON ANTENNAS AND PROPAGATION Best Application Paper Award (H. A. Wheeler Award) in 1989 and 1994, respectively. In 2001, he received the Award of Distinguished Technical Communication from the Society for Technical Communication, USA, and the Science and Technology Progress Award from Hangzhou, China. He is an Associate Editor of several journals and magazines, such as Electromagnetics, IEEE ANTENNAS AND PROPAGATION MAGAZINE, IEEE ANTENNAS AND WIRELESS PROPAGATION LETTERS, and Asian Information-Science-Life. 